\title{
Mean Mutual Information Based Adaptive Modulation and Coding Mechanism for Cooperative Relaying in Wireless Systems
}

\author{
VARGA Mihály $^{1, \mathrm{a}}$, BADIU Mihai-Alin, ${ }^{2, \mathrm{~b}}$, and BOTA Vasile ${ }^{1, \mathrm{c}}$ \\ ${ }^{1}$ Technical University of Cluj-Napoca, 15 Constantin Daicoviciu str. 400020 Cluj - Napoca, Romania \\ ${ }^{2}$ Department of Electronic Systems, Aalborg University, 9220 Aalborg, Denmark \\ àihaly.Varga@com.utcluj.ro, b in_mba@es.aau.dk, ${ }^{\mathrm{c} V}$ asile.Bota@com.utcluj.ro
}

Keywords: Cooperative wireless networks, cross layer design, link adaptation

\begin{abstract}
We propose a link adaptation algorithm for cooperative transmissions in an OFDMAbased wireless system. The link adaptation method selects the transmission type (cooperative or noncooperative), the modulation orders and amount and type of redundancy required to ensure a target block error rate at each phase of the cooperative transmission. The above parameter-values are chosen so as to minimize the number of occupied time-frequency radio resources required to transmit the Kbit log data block. The algorithm employs a BLER performance prediction method based on mutual information. The proposed link adaptation algorithm has linear complexity, but still provides a very good performance, as shown by simulation results.
\end{abstract}

\section{Introduction}

In recent years, wireless services applied in communication, entertainment, healthcare, enterprise, commerce etc. gained vast popularity, mainly due to technological advances. However, the demand for mobile services is increasing at a rapid pace, leading to an exponential increase in traffic amount, as forecasted in [1]. Services with different content types such as video, voice, web/data and audio produce a mixed traffic with various quality of service (QoS) requirements in terms of bit rates, delays and packet-loss. Hence, as also described in [2], future wireless networks should be capable to provide heterogeneous QoS in order to support new type of traffics, such as next generation multimedia services, machine-to machine communications etc. Since a reasonable fairness among an increasing number of users is required, the problem of heterogeneous QoS provisioning over wireless networks is definitely nontrivial to solve in a cost-effective way.

In current cellular networks, the user experience depends on the relative position to the base station, leading to significant performance drop towards cell-edge. However, next generation systems should ensure an experience similar to broadband internet connections independently of the position in the cell. Since improvements in radio link techniques are not very instrumental in solving this propagationrelated problem, cooperative communications gained a lot of attention from the research community in academia and industry. This concept shifts the classical paradigm of point-to-point 'egoistic' communication links towards the collaboration between wireless nodes to achieve common goals [3]. One possible form of cooperation at the physical layer is relaying over intermediate transceivers (relay nodes); by reducing path-loss and combating fading, multi-hop and cooperative relaying represent viable solutions to increase system capacity and/or extend coverage [4].

In a cooperative system, the signals transmitted by the source and the relay are jointly decoded at destination; therefore, a communication link becomes a composite one, since it consists of both the direct and the relay links. When OFDM transmissions are employed, the receiver's decoding performance is affected by the channel realizations over the occupied time-frequency resources in each component link, because the instantaneous signal-to-noise ratio (SNR) varies both across the subcarriers and in time. The link adaptation mechanism becomes more complex, because, given the current states of the constituent channels, it has to decide whether to use the relay or not -- relay activation -and to select the coding rates and modulations to be used on the component links in order to provide the highest bit rate, while ensuring a block error rate (BLER) below a target value. 
To provide a high system performance, the link adaptation algorithms need an accurate method to predict the BLER performance of a transmission on a given realization of the multipath channel, for a particular choice of the modulations and code rate. Performance prediction methods based on mutual information (MI) metrics were shown to ensure a very good prediction accuracy [7] but they were used to obtain effective adaptive modulation and coding algorithms for traditional non-cooperative systems. In [8], the authors of this paper proposed accurate BLER prediction methods for relay-aided communications by also employing mutual information metrics.

In this paper, we propose and evaluate a link adaptation algorithm for an OFDMA system which employs cooperation through distributed turbo coding. Our algorithm provides the relay activation decision, the modulation orders on each occupied resource, and the coding rate (the information block length results implicitly).Simulation results show that the performance in terms of spectral efficiency is very good, thus emphasizing the benefit of cooperative relaying, especially at low and medium SNR values.

\section{System Model}

We consider the downlink transmission in a cell of a cooperative wireless system, where the transmission between one source $(S)$ and one destination $(D)$ node, which in our case are the base station and a user terminal, respectively, can be enhanced by a single relay node $(R)$.

A relay node can serve multiple communication pairs, but on orthogonal resources assigned by a centralized scheduler. The design of the scheduling algorithm is out of the scope of this paper. The considered relays are working in half-duplex mode,therefore, the cooperation protocol involves two time-multiplexed phases, namely the broadcast phase $(B P)$ and the relaying phase $(R P)$.

Each node is equipped with a single antenna, and transmissions are performed using an orthogonal frequency-division multiple access (OFDMA) scheme, i.e. over rectangular time-frequency resources, called radio resource units (RRU) from now on. We also assume in-band relay reception/transmission, such that the relays receive and transmit over a reserved subset of the RRUs that are available in the cell. The sub-carriers are modulated using $\mathcal{M}$-QAM modulations. For any transmission we impose the same modulation order to be used for the RRUs allocated to a user, employed during the same transmission frame. This condition applies for each of the broadcast and relaying-phase, noting that it is possible the modulation orders to differ between the two phases.

The relay processing is done according to the decode-and-forward protocol based on convolutional turbo codes (CTC) [9], as the one described in [10]. The coded block that enters the destination's decoder is constructed in a distributed manner, as follows:

- During the broadcast phase, the source node encodes $K$ information bits using a turbo code with mother code rate $\mathrm{Rm}$. The bits of the obtained mother codeword are interleaved, interlaced [9] and stored on a circular buffer. The rate matching algorithm [9] reads from the circular buffer $L_{B P}=K / R_{B P}$ bits, such that a desired coding rate on the broadcast phase $R_{B P}$ is obtained. The block of length $L_{B P}$ bits is then broadcasted towards the relay node and destination node, over the $S-D$ and $S-R$ channels respectively. The relay decodes the received block and checks the integrity of the decoded data (e.g. via Cyclic Redundancy Check - CRC). The destination node extracts and stores the log-likelihood ratios (LLRs)corresponding to broadcasted bits.

- During the relaying phase the relay re-encodes the information block using the same mother CTC as the source, then, from the circular buffer created for the rate matching algorithm, it selects a number of bits, $L_{R P}$, which are transmitted over the $R-D$ channel. The destination node extras the LLRs of the bits received during this phase, properly re-constructs the LLR flow corresponding to the codeword received during the two phases over two different links (involving different channels). The obtained LLR flow is then fed to the turbo decoder, which also performs the decoding and checks the correctness of the decoded block. 


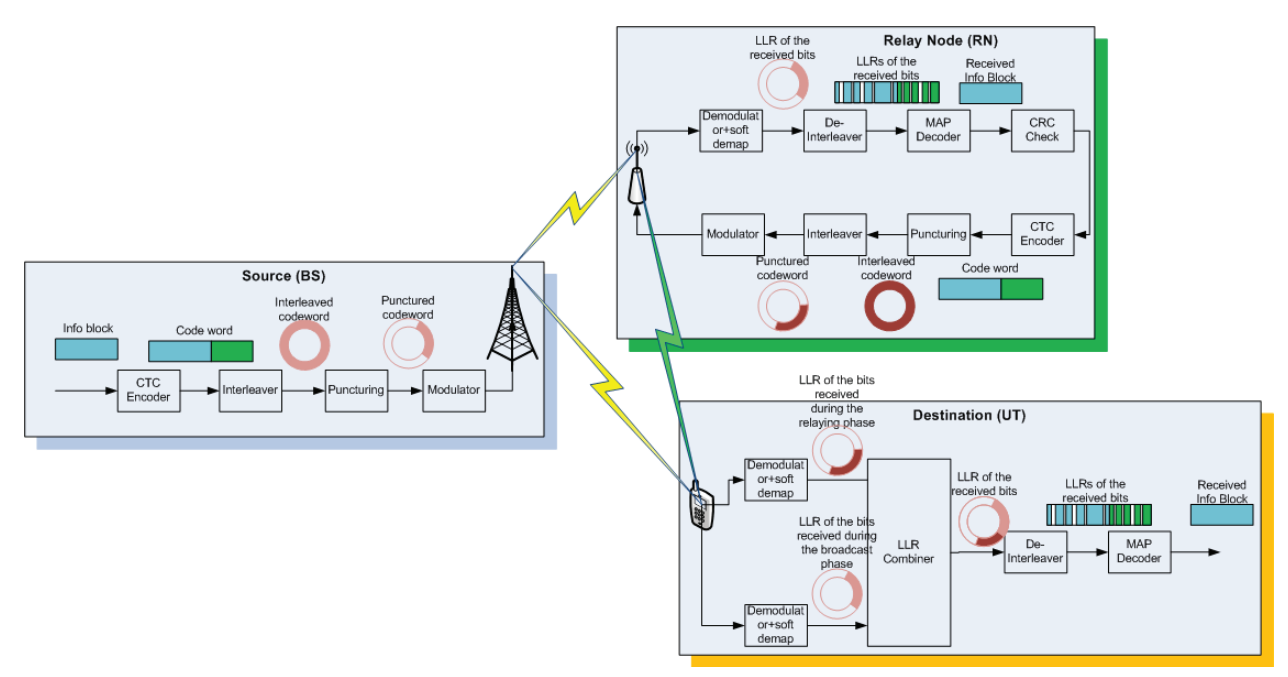

Fig. 1: The block diagram of the considered relay enhanced transmission

Note that the mother codeword obtained by re-encoding at the relay node is identical to the one obtained by the source CTC coder, but the rate matching algorithm at the relay node can select a different set of bits by reading the bits from the circular buffer with a given offset. Depending on the offset value, three redundancy types can be obtained regarding the additional set of bits sent by the relay:

- a totally different new set of bits (incremental redundancy),

- the same set of bits (repetition coding), or

- partial repetition, i.e. only some of the bits sent by the source are repeated (hybrid).

It is important to note that not all transmissions need to be cooperative. A relay is used only if the broadcasted message was successfully decoded at the relay node, and the cooperative transmission (relay enhanced) would provide a greater spectral efficiency than the direct (non-cooperative) sourcedestination transmission. In this paper, this decision of choosing whether to use the cooperative or direct transmission is referred to as relaying phase activation and is realized at each frame by the link adaptation mechanism based on short-term channel state information.

Denoting by $x_{\alpha-\beta}$ a modulation symbol transmitted by node $\alpha \in\{S, R\}$ to node $\beta \in\{R, D\}$, the symbol $y_{\beta}$ received by node $\beta$ is:

$$
y_{\beta}=h_{\alpha-\beta} \cdot \sqrt{\gamma_{\alpha-\beta}} \cdot x_{\alpha-\beta}+n_{\beta}
$$

where $h_{\alpha-\beta}$ denotes the channel coefficient between nodes $\alpha$ and $\beta, \gamma_{\alpha-\beta}$ is the long-term average signal-to-noise ratio of the link $\alpha-\beta$ and $n_{\beta}$ is the complex additive white Gaussian noise (AWGN) with unity power at node $\beta$. Because we consider fixed relay nodes, we assume that the source - relay channel is not subject to the fading phenomenon, i.e. $h_{S-R}=1$. On the other hand, since the destination nodes are mobile, the source-destination and relay-destination channels are assumed to be affected by fading. Assuming that, the channel allocated to one user by the radio resource management algorithm, consist of an adjacent group of time-frequency resources with random beginning position on each radio frame, we consider that the channel coefficients affecting the transmissions are uncorrelated. We also assume that the bandwidth and the time duration of a resource block allocated to one user is sufficient small to neglect the variation of the channel coefficients across the sub-carriers. We adopt a block-fading model, such that each channel has constant coefficients over its sub-carriers during a frame period, but these coefficients vary randomly from one frame to another. Thus, $h_{S-D}$ and $h_{R-D}$ are independent identically distributed (i.i.d.) circularly symmetric complex Gaussian variables with unity power and distribution $\mathcal{C N}(0,1)$. We also assume that every node has perfect instantaneous channel state information. 


\section{Adaptive Modulation and Coding Mechanism}

This section describes the proposed link adaptation mechanism. The main idea is to associate a desired outage probability to each type of service. The task of the link adaptation is to set the transmission parameters so as to guarantee this target by employing the smallest amount of radio resources. This approach applies to each type of supported service by only changing the outage probability.

The link adaptation mechanism uses the instantaneous conditions of the component channels to decide the employment of the cooperative or direct transmission, i.e., relaying phase activation, and to perform adaptive modulation and coding (AMC).

In particular, the AMC mechanism provides the values for the following parameters:

- the number of coded bits sent during the different phases of the transmission $\left(L_{\text {direct }}\right.$ for a direct transmission, $L_{B P}$ and $L_{R P}$ for the broadcast and relaying phases of a cooperative transmission).

- the coding rates corresponding to each transmission phase, and

- the QAM modulation orders of the RRUs used by each transmission.

The above parameter-values are chosen so as to minimize the number of used time-frequency radio resources required to transmit the $K$-bit long data block, while satisfying the block error rate constraints.

To satisfy the BLER targets, the algorithm needs to estimate the performance provided by the multi-link configurations, and to select the most appropriate one. For this, the algorithm employs a BLER performance prediction method based on mutual information. There are several approaches in literature, among which the one proposed in [11], that uses mutual information metrics, and was considered to be the most appropriate for our scenario. In [11] was shown that the BLER performance of CTC codes with coding rate $R c$ and information block length $K$ over fading channels can be estimated, with an arbitrary good precision, by using the mean mutual information per coded bit $(\overline{M I})$ as quality metric to assess the AWGN performance of the CTC codes [11]:

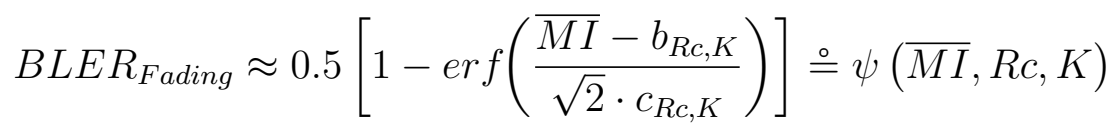

where $\overline{M I}$ is the mean mutual information per coded bit, and $b_{R c, K}$ respectively $c_{R c, K}$ are some coefficients whose values depend on $R c$ and $K$. The values of these coefficients can be determined by computer simulations and curve fitting. The $\operatorname{erf}(\cdot)$ is the error function. The same reference defines $\overline{M I}$ as:

$$
\overline{M I}_{\alpha-\beta}=\frac{\sum_{n=1}^{N} \sum_{p=1}^{m(n)} I_{m(n), p}\left(\rho_{\alpha-\beta}\right)}{\sum_{n=1}^{N} \log _{2}(m(n))}
$$

where: $N$ is the number of employed QAM symbols $x_{\alpha-\beta}, m(n)$ is the modulation order (number of points in constellation) of the $n^{t h}$ QAM symbol, $I_{m(n), p}\left(\rho_{\alpha-\beta}\right)$ is the mutual information between the input bit of the $m(n)$-QAM mapper and the output LLR for the $p^{t h}$ position in the QAM symbol, and $\rho_{\alpha-\beta}$ is the instantaneous SNR of the component link $\alpha-\beta$ expressed by:

$$
\rho_{\alpha-\beta}=\left|h_{\alpha-\beta}\right|^{2} \cdot \gamma_{\alpha-\beta}
$$

In our system model, since it was assumed the signal $x_{\alpha-\beta}$ is transmitted using the same modulation order $m(n)=m_{\alpha-\beta}$; for $n=\overline{1 . . N}$ on each RRU assigned to one user during a transmission phase, the expression Eq. 3 can be rewritten as Eq. 5:

$$
\overline{M I}_{\alpha-\beta}^{m_{\alpha-\beta}}=I_{m_{\alpha-\beta}}\left(\rho_{\alpha-\beta}\right)
$$


where $I_{m}(\cdot)$ maps the SNR to the mutual information domain and, for each modulation order, can be approximated with analytical functions, [11].

In order to select the appropriate modulation order and coding rate which ensure a transmission with a maximum allowed block error rate over the given channel we need the inverse function of $\psi(\cdot)$, which according to Eq. 2 can be expressed as:

$$
\overline{M I}(R c, K, B L E R)=\sqrt{2} \cdot c_{R c, K} \cdot \operatorname{erf}^{-1}(1-2 \cdot B L E R)+b_{R c, K}
$$

The drawback of the above described method is that for an accurate prediction it is necessary to determine and store the value of the $b_{R c, K}$ and $c_{R c, K}$ coefficients for each value of $K$ and $R c$. To overcome this drawback we use a property of the CTC codes noticed during our studies: for the same $K$ and for two different coding rates, the distance between the two BLER curves represented in the $\overline{M I}$ domain can be very well approximated as constant at any BLER value. We propose an empirical analytic function to determine the $\overline{M I}$ value necessary to ensure a given BLER, for an information block length of $K$ bits encoded with coding rate $R c$ :

$$
\overline{M I}(R c, K, B L E R)=\lambda_{K} R c^{2}+\mu_{K} R c+\overline{M I}(R m, K, B L E R)
$$

where variables $\lambda_{K}$ and $\mu_{K}$ have constant values for a given $K$, and can be determined by computer simulations and curve fitting. $\overline{M I}(R m, K, B L E R)$ is the particular $\overline{M I}$ value which provides a specific BLER value for a coding rate equal to the mother rate $R m$, and should be computed based on Eq. 6. Using this approximation, it is sufficient to determine and store for all possible value of $K$ the 4-tuple $\left(b_{R m, K}, c_{R m, K}, \lambda_{K}, \mu_{K}\right)$.

Next, we apply the above methodology to derive the steps required by the link adaptation mechanism to perform AMC and decide relaying phase activation. First, we determine the minimum number of resources, measured in RRUs which actually could be expressed in number of QAM-symbols, required by a direct transmission $\left(N_{\text {direct }}\right)$ and by a cooperative one $\left(N_{\text {coop }}\right)$. The scheme which offers the smallest number will be selected for the initial transmission.

To determine the minimum amount of resources required by a direct transmission, first we determine the minimum length of a coded block which ensures the imposed BLER if the transmission over $S-D$ channel uses a modulation with order $m_{S-D}$. Denoting by $\rho_{S-D}$ the instantaneous signal to noise ratio of the $S-D$ link, the length of the coded block required to transmit $K$ information bits using a direct transmission is:

$$
L_{\text {direct }}=\Lambda_{\text {direct }}^{m_{S-D}}\left(\rho_{S-D}, K, p_{e}^{0}\right)
$$

In the above expression, the function $\Lambda_{\text {direct }}^{m_{S-D}}\left(\rho_{S-D}, K, p_{e}^{0}\right)$ computes the shortest CTC codeword length, so that when transmitting $K$ information bits using a modulation with order $m_{S-D}$ over a channel with effective SNR $\rho_{S-D}$, the block error rate will be at most $p_{e}^{0}$. In order to derive the closed form of function $\Lambda_{\text {direct }}^{m_{S-D}}\left(\rho_{S-D}, K, p_{e}^{0}\right)$, we compute first the highest coding rate $R c_{m_{S-D}}$ which ensures the imposed BLER, if a modulation with order $m_{S-D}$ is used over the $S-D$ link. $R c_{m_{S_{D}}}$ can be obtained by solving Eq. 7, and equals:

$$
R c_{m_{S-D}}=\frac{-\mu_{K}+\sqrt{\mu_{K}{ }^{2}-4 \lambda_{K}\left(\overline{M I}\left(R m, K, p_{e}^{0}\right)-\overline{M I}_{S-D}^{m_{S-D}}\right)}}{2 \cdot \lambda_{K}}
$$

Taking into account that $R c_{m_{S-D}}=K / L$ the expression of $\Lambda_{\text {direct }}^{m_{S-D}}\left(\rho_{S-D}, K, p_{e}^{0}\right)$ is:

$$
\Lambda_{\text {direct }}^{m_{S-D}}\left(\rho_{S-D}, K, p_{e}^{0}\right)=\left\lceil\frac{K \cdot 2 \cdot \lambda_{K}}{-\mu_{K}+\sqrt{\mu_{K}^{2}-4 \lambda_{K}\left(\overline{M I}\left(R m, K, p_{e}^{0}\right)-\overline{M I} \bar{I}_{S-D}^{m_{S-D}}\right)}}\right\rceil
$$

The transmission of the $L_{\text {direct }}$ coded bits requires a total of $N_{\text {direct }}$ QAM symbols:

$$
N_{\text {direct }}=\min _{m_{S-D} \in \mathcal{M}}\left(\frac{\Lambda_{\text {direct }}^{m_{S-D}}\left(\rho_{S-D}, K, p_{e}^{0}\right)}{\log _{2}\left(m_{S-D}\right)}\right)
$$


where $\mathcal{M}$ is the set of available modulation orders. Also, the coding rate of the direct transmission is $R c_{\text {direct }}=K / L_{\text {direct }}$.

To determine the minimum amount of resources required by a cooperative transmission, one has to take into account that the relay node has to be able to decode the broadcast transmission. For this, the number of resources used during the broadcast phase $N_{B P}$ should be high enough, so that the relay will decode with a block error probability at most $p_{R}$. For a modulation with order $m_{S-D}=m_{S-R}=m_{B P}$, the minimum number of resources $N_{\text {relay }}^{m_{B P}}$ required to satisfy the BLER constraint at the relay should be computed using Eq. 11, where $\rho_{S-D}$ and $p_{e}^{0}$ are replaced by $\rho_{S-R}$ and $p_{R}$, respectively. Of course, $N_{B P}$ is upper limited by $N_{\text {direct }}$, otherwise the cooperative transmission would not be worthwhile.

As described in section II, the relay's message contains two types of bits:

- a set of bits that are different from the ones transmitted by S; these provide coding gain for the $\mathrm{CTC}$, and their $\overline{M I}$ is averaged with the one of the bits sent by $\mathrm{S}$;

- a set of bits that have already been transmitted by $S$; these repeated bits improve their reliability by exploiting the energy gain and the diversity. The method proposed in [8] is employed here to compute the mean mutual information of the repeated bits $\overline{M I}_{r e p}$; this value is given by Eq. 12, in which Eq. 5 and its inverse are used for BPSK modulation.

$$
\overline{M I}_{\text {rep }}=I_{2}\left(I_{2}^{-1}\left(\overline{M I}_{S-D}^{m_{B P}}\right)+I_{2}^{-1}\left(\overline{M I}_{R-D}^{m_{R-D}}\right)\right)
$$

Thus, after the relaying phase, the "accumulated" mutual information can be calculated as:

$$
\overline{M I}_{\text {coop }}=\frac{\overline{M I}_{S-D}^{m_{B P}} \cdot\left(L_{B P}-L_{r e p}\right)+\overline{M I}_{R-D}^{m_{R-D}} \cdot\left(L_{R P}-L_{r e p}\right)+L_{r e p} \cdot \overline{M I}_{r e p}}{L_{B P}+L_{R P}-L_{r e p}}
$$

where $L_{r e p}$ is the number of coded block bits repeated in both phases of the initial transmission, and whose values range between the following thresholds:

$$
\max \left(0 ; L_{B P}+L_{R P}-K \cdot\left(R_{m}\right)^{-1}\right) \leq L_{r e p} \leq \min \left(L_{B P} ; L_{R P}\right)
$$

The $\left(L_{R P}, L_{r e p}\right)=\Lambda_{\text {coop }}^{m_{R-D}}\left(M I_{S-D}^{m_{B P}}, K / L_{B P}, \rho_{R-D}, K, p_{e}^{0}\right)$ function computes the minimum number of $L_{R P}$ bits sent during the relaying phase, among which a number of $L_{\text {rep }}$ bits are repeated. To compute the necessary codeword length and the number of repeated bits in the broadcast phase of the cooperative transmission, we can write an inequality similar to Eq. 7. :

$$
\overline{M I}_{\text {coop }} \geq \lambda_{K} \cdot R c_{\text {coop }}{ }^{2}+\mu_{K} \cdot R c_{\text {coop }}+\overline{M I}\left(R m, K, p_{e}^{0}\right)
$$

where $\overline{M I}_{\text {coop }}$ and $R c_{\text {coop }}$ are the mean mutual information and the coding rate, respectively, at the input of the receiver's CTC decoder. $R c_{\text {coop }}$ can be expressed as:

$$
R c_{\text {coop }}=K \cdot\left(L_{B P}+L_{R P}-L_{\text {rep }}\right)^{-1}
$$

In Eq. 13 and Eq. $16 L_{R P}$ is the total number of bits which will be sent during the relaying phase of the transmission, $L_{r e p}$ is the number of repeated bits $\overline{M I}_{R P}^{m_{R-D}}$ is the mean mutual information of the $L_{R P}$ bits and $\overline{M I}_{r e p}$ is the mean mutual information of the repeated bits, computed using Eq. 12.

Substituting Eq. 12 and Eq. 16 in Eq. 15, we get:

$$
\begin{gathered}
\frac{\overline{M I}_{S-D} \cdot\left(L_{B P}-L_{r e p}\right)+\overline{M I}_{R-D}^{m_{R-D}} \cdot\left(L_{R P}-L_{r e p}\right)+L_{r e p} \cdot \overline{M I}_{r e p}}{L_{B P}+L_{R P}-L_{r e p}} \geq \\
\geq \frac{\lambda_{K} \cdot K^{2}+\mu_{K} \cdot K \cdot\left(L_{B P}+L_{R P}-L_{r e p}\right)}{\left(L_{B P}+L_{R P}-L_{r e p}\right)^{2}}+\overline{M I}\left(R m, K, p_{e}^{0}\right)
\end{gathered}
$$

The function $\Lambda_{\text {coop }}^{m_{R-D}}\left(\overline{M I}_{S-D}, R c_{B P}, \rho_{R-D}, K, p_{e}^{0}\right)$ is the pair $\left(L_{R P}, L_{r e p}\right)$ which satisfies (17) and minimizes $L_{R P}$. Solving (17) in terms of $L_{R P}$ and choosing $L_{r e p}$ so that to minimize $L_{R P}$ we get:

$$
\Lambda_{\text {coop }}^{m_{R-D}}\left(\overline{M I}_{S-D}, R c_{B P}, \rho_{R-D}, K, p_{e}^{0}\right)=\min _{L_{r e p}}\left(\frac{-L_{r e p} \cdot \kappa_{1}-\kappa_{2}+\sqrt{L_{r e p}^{2} \cdot \kappa_{3}+L_{r e p} \cdot \kappa_{4}+\kappa_{5}}}{2 \cdot \kappa_{6}}\right)
$$


where:

$$
\begin{aligned}
\kappa_{1} & =\overline{M I}_{S-D}+2 \cdot \overline{M I}_{R-D}^{m_{R-D}}-\overline{M I}_{r e p}-\overline{M I}\left(R m, K, p_{e}^{0}\right) \\
\kappa_{2} & =L_{B P}\left(2 \cdot \overline{M I}\left(R m, K, p_{e}^{0}\right)-\overline{M I}_{S-D}\right)-L_{B P} \overline{M I}_{R-D}^{m_{R-D}}+\mu_{K} \cdot K \\
\kappa_{3} & =\kappa_{1}^{2}-4 \cdot \kappa_{6} \cdot\left(\overline{M I}\left(R m, K, p_{e}^{0}\right)+\overline{M I}_{r e p}\right)+4 \cdot \kappa_{6} \cdot\left(\overline{M I}_{S-D}+\overline{M I}_{R-D}^{m_{R-D}}\right) \\
\kappa_{4} & =2 \kappa_{1} \cdot \kappa_{2}-4 \kappa_{6} \cdot\left(L_{B P}\left(2 \overline{M I}_{S-D}-2 \overline{M I}\left(R m, K, p_{e}^{0}\right)+\overline{M I}_{R-D}^{m_{R-D}}-\overline{M I}_{r e p}\right)-\mu_{K} K\right) \\
\kappa_{5} & =\kappa_{2}^{2}-4 \cdot \kappa_{6} \cdot \lambda_{K} \cdot K^{2}-4 \cdot \kappa_{6} \cdot\left(L_{B P} \mu_{K} \cdot K+L_{B P}{ }^{2} \cdot \overline{M I}\left(R m, K, p_{e}^{0}\right)\right) \\
\kappa_{6} & =\overline{M I}\left(R m, K, p_{e}^{0}\right)-\overline{M I}_{R-D}^{m_{R-D}}
\end{aligned}
$$

When modulation order $m_{R-D}$ is employed during the relaying phase, the number of resources of the R-D channel used to ensure the target block error probability is:

$$
N_{R P}^{m_{R-D}}=\left\lceil L_{R P} \cdot \log _{2}\left(m_{R-D}\right)^{-1}\right\rceil
$$

Finally, the number of resources required by a cooperative transmission is:

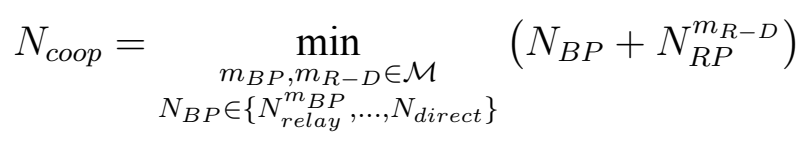

The most spectrally-efficient configuration ensures at the destination node the imposed BLER, by using the fewest number of resources. The minimum number of resources $N_{0}$ used during the transmission will be:

$$
N_{0}=\min \left(N_{\text {direct }}, N_{\text {coop }}\right)
$$

Note that the minimum number of used resources depends on the instantaneous conditions of the component channels and on the imposed BLER values at the relay and destination. The configuration that minimizes Eq. 27 will be the result of the relaying phase activation decision, while the parameters that led to that value are the output of the AMC algorithm.

\section{Numerical Results}

In order to gain more insights into the performance and behaviour of the considered link adaptation mechanism, we provide in this section some numerical results based on the analytical model derived in the previous sections.

As previously mentioned, the spectral efficiency of the proposed protocol depends on the longterm average signal to noise ratios (SNR) experienced by the involved nodes on the component links. In order to obtain a distribution of the 3-tuple of average SNRs $\left(\gamma_{S-R}, \gamma_{S-D}, \gamma_{R-D}\right)$, we assume a hexagonal cell of radius $r$ having the base station in its center. We consider a number of $n_{R}$ fixed relay nodes, uniformly distributed around the base station on a circle of radius $r_{R}<r$. In this paper we assumed $r=1$ kilometer and $n_{R}=6$ relays positioned at $r_{R}=r / 3$ from the base station.

The transmission scheme is an OFDM one with $N_{F F T}=1024$, subcarrier spacing of $15 \mathrm{kHz}$, and chunk dimensions $N_{f}=12$ subcarriers and $N_{t}=7$ OFDM symbols. The ITU Pedestrian A channel model is used.

We also assume that the average SNR is affected only by free-space path-loss, i.e., we do not take into account the shadowing and the inter-cell interferences, and the noise has constant power spectral density across the cell. In this case, the long-term average SNR of a link will depend on the transmitted power, the distance between the transmitter and receiver, and the noise power in the transmission band. Taking into the account that the power of band-limited noise is constant in the cell, the SNR at the receiver can be expressed function of the SNR at the transmitter side as:

$$
10 \lg \left(\gamma_{\alpha-\beta}\right)=10 \lg \left(\gamma_{\alpha}\right)-\left(130.19+37.6 \lg \left(d_{\alpha-\beta}\right)\right)
$$


where $\gamma_{\alpha}$ is the average signal to noise ratio at the transmitter $\alpha \in\{S, R\}$, and $d_{\alpha-\beta}$ is the distance in kilometres from the transmitter to the receiver. The second term of Eq. 28 is the path-loss model for an urban or suburban scenario, as considered in [13] for a carrier frequency of $2.5 \mathrm{GHz}$. In this paper, we set the value for $\gamma_{\alpha}$ so that the smallest $10 \lg \left(\gamma_{\alpha-\beta}\right)$ at the cell edge to be $0 \mathrm{~dB}$.

Since the relay nodes can be positioned as desired, the average SNR of the S-R channel can be ensured to be significantly higher than the average SNRs of $S-D$ and $R-D$ channels, e.g., by equipping the relay nodes with antennas dedicated for the communications with the base station. For each possible position of a destination node in the cell, we consider that the nearest relay node is assigned to that destination node. We also assume all nodes have omni-directional transmit antennas with $0 \mathrm{~dB}$ antenna gain.

The accuracy of the above BLER prediction methodology is proven by Fig. 2, and Fig. 3 which presents the simulated vs.predicted BLER performance of the CTC with parameters specified in [9] for several values of $K$ and coding rates for AWGN and frequency selective fading channels.

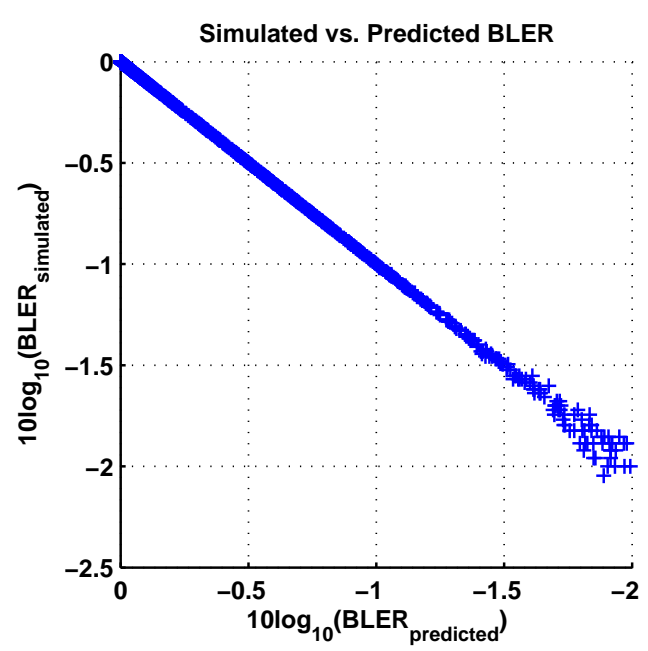

Fig. 2: Simulated vs. Predicted BLER in AWGN channel

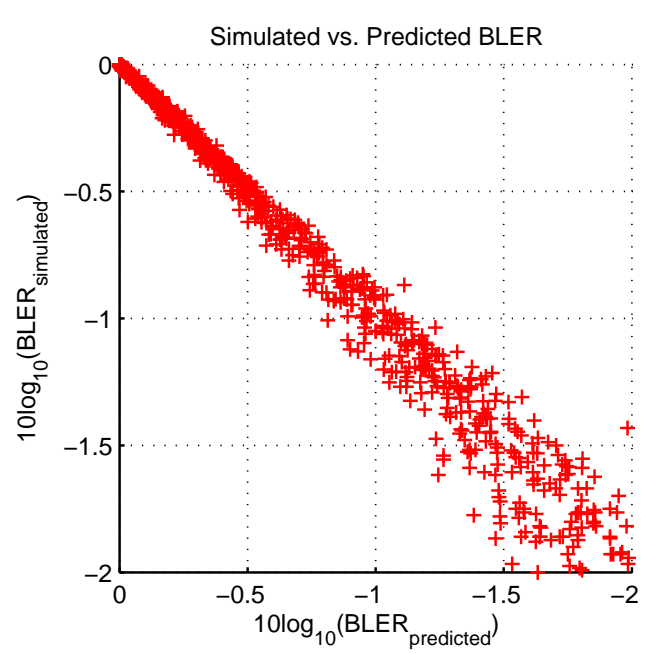

Fig. 3: Simulated vs. Predicted BLER in frequency selective mobile channel

Fig. 4 show the resulted spectral efficiencies in information bits correctly decoded per QAM symbol vs. the average SNR of the direct link. As reference, the capacity of a 64QAM transmission over a Rayleigh faded channel is plotted. Fig. 5 illustrates the variation of the spectral efficiency with the long-term average SNR of the $S-D$ and $R-D$ links, ensured by a cooperative transmission which uses the proposed link adaptation algorithm, having an imposed outage probability $p_{e}^{0}=0.001$. Fig. 6 shows the variation of the spectral efficiency with average SNR of the direct $S-D$ link, for $p_{e}^{0}=\left\{10^{-1}, 10^{-1.33}, 10^{-1.66}, 10^{-2}\right\}$. To point out the influence of the $R-D$ channel quality, the figure presents the curves for three cases: $\gamma_{R-D}=\gamma_{S-D}, \gamma_{R-D}=\gamma_{S-D}+6 d B$ and $\gamma_{R-D}=\gamma_{S-D}+12 d B$ (three slices of Fig. 5). The following facts can be noticed:

- significant improvements of up to 1-1.5 bit/symbol (corresponding to a gain up to 6 -- $8 \mathrm{~dB}$ ) is brought by cooperation at low-medium SNR values, depending on the quality of the $R-D$ channel;

- as expected, the performance gain increases with the quality of the $R-D$ channel;

- the spectral efficiency of the direct transmission is within 2-3 dBs from the reference curve.

The plot illustrating the distribution in the cell of the differences between the spectral efficiencies ensured by the cooperative and direct transmissions (Fig. 7) show that the relay enhanced transmission brings a $0.5-0.9$ bits/QAM symbol gain in spectral efficiency in a large part of the cell. Fig. 7 also show that the cooperative transmission is used in the vicinity of the relay nodes and at the edge of the cell. Note that as the SNR of the direct link increases, the cooperation ratio decreases. For high SNR values, the algorithm automatically selects the chunks from the direct link, i.e. the relay is not activated. 


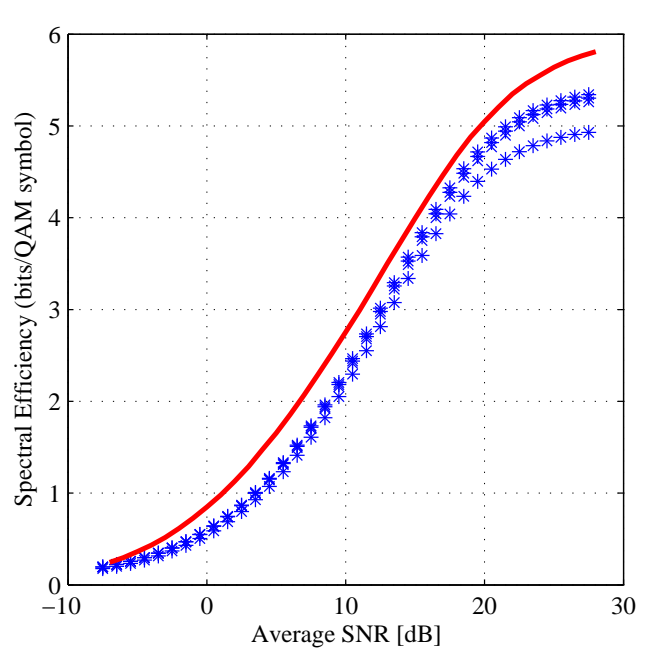

Fig. 4: Variation of spectral efficiency vs. average SNR (stars) ensured by the direct connection (continuous line - the capacity of the 64-QAM over Rayleigh channel)

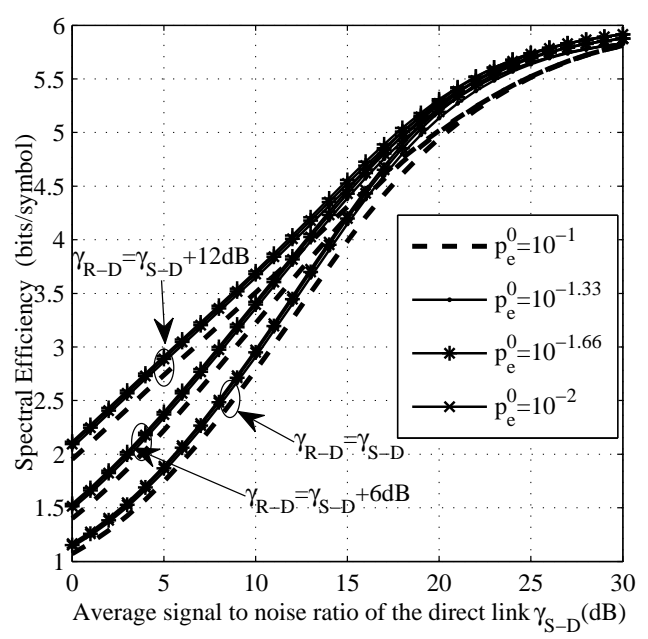

Fig. 6: Variation of spectral efficiency vs. average SNR of the direct link

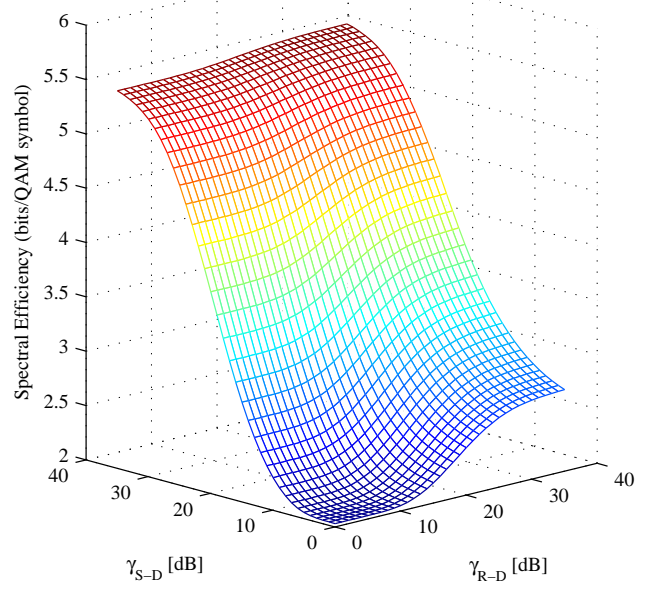

Fig. 5: Variation of spectral efficiency vs. average SNR of the component links, in case of relay enhanced (cooperative) transmission (in this case the target outage probability is $\left.p_{e}^{0}=0.001\right)$

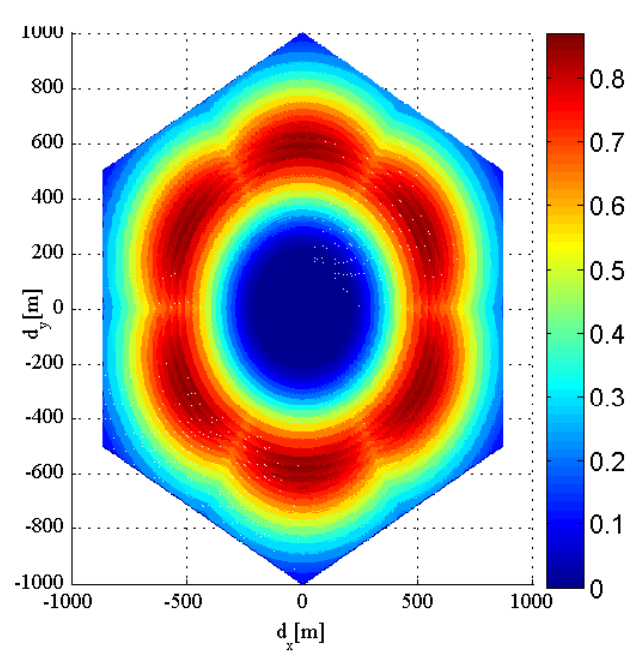

Fig. 7: Difference between the spectral efficiency ensured by the cooperative and non-cooperative transmissions (bits/QAM symbols)

\section{Conclusion}

This paper proposed a link adaptation algorithm for OFDMA systems employing a distributed coding scheme. The algorithm also automatically performs relay activation by selecting the most efficient scheme (direct or cooperative), fact that introduces a new dimension of link adaptation, specific to cooperative transmissions.

The proposed algorithm uses a block error rate estimation method, based on mutual information, to predict the block error rate provided by a direct/cooperative transmission with mixed modulation orders over multipath fading channels. The modulation orders and message lengths are selected so as to maximize the spectral efficiency and, in the same time, observing the block error rate constraints.

The results for direct transmissions are close to the reference curve, showing a very good performance of the link adaptation algorithm. Moreover, the spectral efficiency is significantly improved by cooperation, at low and medium SNR values on the direct S-D link. 


\section{Acknowledgement}

The work of M. Varga was supported by the project "Development and support of multidisciplinary postdoctoral programmes in major technical areas of national strategy of Research - Development - Innovation" 4D-POSTDOC, contract no. POSDRU/89/1.5/S/52603, project co-funded by the European Social Fund through Sectoral Operational Programme Human Resources Development 2007-2013.

\section{References}

[1] "Cisco Visual Networking Index: Global Mobile Data Traffic Forecast Update, 2010 - 2015," Cisco Systems, Inc., White Paper, February 2011.

[2] "Requirements related to technical performance for IMT-Advanced radio interface(s)," International Telecommunication Union, Report M.2134, December 2008.

[3] H. P. Frank and F. Katz, Cooperation in Wireless Networks: Principles and Applications: Real Egoistic Behavior Is to Cooperate! Secaucus, NJ, USA: Springer-Verlag New York, Inc., 2006.

[4] E. Zimmermann, P. Herhold, G. Fettweis, "On the performance of cooperative relaying protocols in wireless networks," European Transactions on Telecommunications, vol. 16, pp. 5--16, 2005.

[5] Z. Zhang and T. Duman, "Capacity-Approaching Turbo Coding For Half-Duplex Relaying," Communications, IEEE Transactions on, vol. 55, no. 10, pp. 1895 --1906, oct. 2007.

[6] A. Chakrabarti, A. D. Baynast, A. Sabharwal, and B. Aazhang, "Low density parity check codes for the relay channel," Selected Areas in Communications, IEEE Journal on, vol. 25, no. 2, pp. 280 --291, february 2007.

[7] K. Sayana, J. Zhuang, and K. Stewart, "Short term link performance modeling for ML receivers with mutual information per bit metrics," in Proc. GLOBECOM 2008, 2008.

[8] M.-A. Badiu, M. Varga, and V. Bota, "Link performance prediction methods for cooperative relaying in wireless networks," in Wireless Communication Systems (ISWCS), 2010 7th International Symposium on, sept. 2010, pp. 556 --560.

[9] Evolved Universal Terrestrial Radio Access (E-UTRA);Multiplexing and channel coding, 3GPP Standardization Group Std. 3GPP TS 36.212 V9.2.0, June 2010.

[10] D. Castelain, C. Ciochina, A. Silva, A. Gameiro, D. Neves, S. Teodoro, C. Ribeiro, N. Chiurtu, V. Bota, M. Varga, C. M. Bota, Z. A. Polgar, M. P. Stef, M. A. Badiu, and V. Savin, "Final advanced PHY layer algorithm selection and results," CODIV FP7ICT-2007-215477, Deliverable, September 2010. [Online]. Available: http://www.ictcodiv.eu/private/docs/deliverables/D3.3b.pdf

[11] K. Sayana, J. Zhuang, and K. Stewart, "Short Term Link Performance Modeling for ML Receivers with Mutual Information per Bit Metrics," in Global Telecommunications Conference, 2008. IEEE GLOBECOM 2008. IEEE, 30 2008-dec. 4 2008, pp. 1--6.

[12] M.A. Badiu, M. Varga, V. Bota and A. Vlaicu, ' Simplification of a Link Performance Prediction Method Based on Mutual Information," in Acta Technica Napocensis (Electronics and Telecommunications), September 2012.

[13] R. Srinivasan, L. Jallou, R. Novak, and J. Par, "IEEE 802.16m Evaluation Methodology Document (EMD)," IEEE 802.16 Broadband Wireless Access Working Group, Standard IEEE 802.16m-08/004r5, January 2009. 